\title{
Microbial community structure and diversity in a municipal solid waste landfill
}

\author{
Xiaolin Wang ${ }^{\mathrm{a}}$, Aixin Cao ${ }^{\mathrm{b}}$, Guozhu Zhao ${ }^{\mathrm{a}, *}$, Chuanbin Zhou ${ }^{\mathrm{b}, *}$, Rui Xu ${ }^{\mathrm{a}, \mathrm{b}}$ \\ ${ }^{a}$ College of Biological Sciences and Biotechnology, Beijing Forestry University, Beijing 100083, China \\ ${ }^{\mathrm{b}}$ State Key Laboratory of Urban and Regional Ecology, Research Center for Eco-environmental Sciences, Chinese Academy of Sciences, Beijing 100085, China
}

\section{A R T I C L E I N F O}

\section{Article history:}

Received 4 August 2016

Revised 5 April 2017

Accepted 9 April 2017

Available online 22 April 2017

\section{Keywords:}

Microbial

Diversity

Structure

MiSeq

Landfill

Municipal solid waste

\begin{abstract}
A B S T R A C T
Municipal solid waste (MSW) landfills are the most prevalent waste disposal method and constitute one of the largest sources of anthropogenic methane emissions in the world. Microbial activities in disposed waste play a crucial role in greenhouse gas emissions; however, only a few studies have examined metagenomic microbial profiles in landfills. Here, the MiSeq high-throughput sequencing method was applied for the first time to examine microbial diversity of the cover soil and stored waste located at different depths $(0-150 \mathrm{~cm})$ in a typical MSW landfill in Yangzhou City, East China. The abundance of microorganisms in the cover soil $(0-30 \mathrm{~cm})$ was the lowest among all samples, whereas that in stored waste decreased from the top to the middle layer $(30-90 \mathrm{~cm})$ and then increased from the middle to the bottom layer $(90-150 \mathrm{~cm})$. In total, 14 phyla and 18 genera were found in the landfill. A microbial diversity analysis showed that Firmicutes, Proteobacteria, and Bacteroidetes were the dominant phyla, whereas Halanaerobium, Methylohalobius, Syntrophomonas, Fastidiosipila, and Spirochaeta were the dominant genera. Methylohalobius (methanotrophs) was more abundant in the cover layers of soil than in stored waste, whereas Syntrophomonas and Fastidiosipila, which affect methane production, were more abundant in the middle to bottom layers $(90-150 \mathrm{~cm})$ in stored waste. A canonical correlation analysis showed that microbial diversity in the landfill was most strongly correlated with the conductivity, organic matter, and moisture content of the stored waste.
\end{abstract}

(c) 2017 Elsevier Ltd. All rights reserved.

\section{Introduction}

Municipal solid waste (MSW) landfills are the most prevalent waste disposal method and constitute one of the largest sources of anthropogenic methane emissions in the world (IPCC, 2014). About $60 \%$ of MSW generated is disposed of in landfills in China and the United States (USEPA, 2009; Zhang et al., 2010), generating 112 and 178 million tons $\mathrm{CO}_{2} \mathrm{eq}$, respectively (NCCC, 2013; USEPA, 2016). Microbial activities in disposed waste play a crucial role in decomposing organic waste and greenhouse gas emissions (Slezak et al., 2015). Various microorganisms proliferate abundantly in landfills due to richness of the organic matter and substrate complexity; therefore, landfills have been considered microbial pools (Song et al., 2015a,b). Many laboratory studies have examined the microbial activities associated with landfills (e.g., in leachate treatment systems, Fernandes et al., 2013) and bioreactor landfills (Sang et al., 2012; Bareither et al., 2013; Lee et al., 2014). However, the site-specific microbial profiles in land-

\footnotetext{
* Corresponding authors.

E-mail addresses: zhaogz@im.ac.cn (G. Zhao), cbzhou@rcees.ac.cn (C. Zhou).
}

fills could differ from those identified in laboratory studies. Only limited research on the microbial profiles in landfills has examined the identity, ecology, and diversity of the microbial community structures at different landfill sites (Gomez et al., 2011; Song et al., 2015a,b). However, none of these studies discussed the vertical distribution of microbial composition in landfills.

Vertical distance from the surface to the bottom of a landfill may reflect different levels of the moisture content and composition of stored waste, and, accordingly, change the abundance and distribution of the microbial community. For example, methanogens and acid-producing bacteria decompose organic matter and generate methane at the bottom of a landfill (Slezak et al., 2015; Wang et al., 2015). Clostridium, Syntrophus, and Sporotomaculum promote methane production by accelerating hydrocarbon degradation in landfills (Gieg et al., 2014). Additionally, methanotrophs (e.g., Methylocystis and Methylocaldum) are able to fix methane using an enzyme complex and methane monooxygenase under aerobic conditions at the surface of landfills (Stralis-Pavese et al., 2004). Therefore, a study of the vertical distribution of microbial communities is needed to determine the microbial niches at each depth in landfills (Dong et al., 2015). 
Several methods have been applied to identify the microbial profiles in landfills, such as terminal restriction fragment length polymorphism (T-RFLP) (Sawamura et al., 2010; Gomez et al., 2011) and polymerase chain reaction-denaturing gradient gel electrophoresis (PCR-DGGE) (Uchida et al., 2009; Song et al., 2015a). However, a previous study that used the T-RFLP and PCR-DGGE techniques was unable to produce a high-resolution microbial profile in landfills (Zhang et al., 2016). Novel high-throughput sequencing techniques (e.g., MiSeq) are an emerging tool to analyze microbial diversity and relative abundance and offer the advantages of high sensitivity and integral coverage on sequencing microorganisms. Thus, these techniques enable characterization of trace bacteria and uncultured microorganisms in a complex environment (Glenn, 2011). However, no study has used MiSeq to analyze the microbial communities in landfills.

In the present study, a typical MSW landfill located in Yangzhou, Jiangsu Province, East China was selected as the case study. Microbial diversity in the cover soil and stored waste was analyzed in different layers $(0-150 \mathrm{~cm})$ of the landfill by applying the MiSeq method at the phylum and generic levels. Our study generated metagenomic datasets for both microbial and environmental studies; the factors (electrical conductivity [EC], organic matter, moisture content, and disposal depth) that potentially influence the microbial structure and diversity in landfills are also discussed.

\section{Materials and methods}

\subsection{Sampling method}

Samples were collected from the Zhaozhuang landfill (latitude: $32.4713^{\circ} \mathrm{N}$, longitude: $119.3189^{\circ} \mathrm{E}$ ), a typical MSW landfill located in Yangzhou, Jiangsu Province, East China, with a humid and subtropical climate. The landfill began operations in August 2002 and now occupies an area of $150,000 \mathrm{~m}^{2}$. It receives about 0.22 million tons of mixed MSW per year. The depth of the cover soil of each site was about $20 \mathrm{~cm}$, and the stored waste was sampled at depths of $0-150 \mathrm{~cm}$, in which the waste was stored for approximately 7 years after closure.

The cover soil and stored waste (both were solid samples) at two sampling sites (Site-1 and Site-2), which were located $10 \mathrm{~m}$ apart in the same landfill cell, were collected for parallel analysis. To examine the vertical distribution of the microbial community in stored waste, we sampled at $30-\mathrm{cm}$ intervals to a depth of $150 \mathrm{~cm}$. In total, we collected 12 samples, labeled $1 \_0,1 \_3,1 \_6$, 1_9, 1_12, 1_15, 2_0, 2_3, 2_6, 2_9, 2_12, and 2_15. Labels 1_ and 2 _ indicates Site 1 and Site 2, respectively; whereas label “_0" refers to the cover soil $(0-20 \mathrm{~cm})$ sample; labels “_3”, “_6”, “_9”, “_12", and "_15" refer to the waste sampled from depths of $0-30$, $30-60,60-90,90-120$, and $120-150 \mathrm{~cm}$, respectively. A shovel was used to excavate a $1.0 \mathrm{~m} \times 1.5-\mathrm{m}$ area in each sampling site. The cover soil and stored waste in each layer were completed removed and transferred to a sterilized plastic sheet, and the shovel was cleaned before excavating a new layer to avoid contamination between layers. Approximately $500 \mathrm{~g}$ of sample was collected at each site by the coning and quartering method, and each sample was placed in an icebox for a physicochemical analysis.

\subsection{Physicochemical analysis}

To test the physicochemical properties, each sample was mixed evenly and sieved through a 100-mesh screen to remove large particles (plastic bags, stone, glass, etc.). A soil-water (1:5, w/v) suspension was prepared 30 min prior to $\mathrm{EC}$ and $\mathrm{pH}$ measurements using the Professional Meter (PP-20, Sartorius, Göttingen, Germany). The concentration of organic matter was determined by
Tyurin's method (Nikolskii, 1963). Moisture content was measured gravimetrically. Cation exchange capacity (CEC) was measured using the sodium acetate exchange method (Rhoades, 1982). Total carbon (TC) and total nitrogen (TN) were tested by an elemental analyzer (vario PYRO cube; Elementar, Langenselbold, Germany). Ammonium in the samples was displaced by $100 \mathrm{~mL}$ of $0.01 \mathrm{M}$ $\mathrm{CaCl}_{2}$ for $60 \mathrm{~min}$. The extract was filtered, and the $\mathrm{NH}_{4}^{+}-\mathrm{N}$ concentration was measured using a continuous flow analytical system (Autoanalyzer 3, Bran + Luebbe: SPX Flow Technology, Norderstedt, Germany).

\subsection{DNA extraction and polymerase chain reaction (PCR) amplification}

To prepare samples for DNA extraction, a 10-mesh screen was used to remove large particles from the stored waste. A fivepoint sampling mode was applied for sub-sampling, and a 1.52.0-g sub-sample was collected for extracting DNA. The samples were stored at $-80^{\circ} \mathrm{C}$ before extraction. DNA in each sample was extracted using a MoBio Power Soil DNA extraction kit (MoBio, Carlsbad, CA, USA). The DNA concentration was quantified on a NanoDrop 2000 spectrophotometer (Thermo Scientific, Wilmington, DE, USA). The V4-V5 region of the microbial $16 \mathrm{~S}$ ribosomal RNA gene was amplified by PCR $\left(95^{\circ} \mathrm{C}\right.$ for $3 \mathrm{~min}$, followed by 27 cycles at $95{ }^{\circ} \mathrm{C}$ for $30 \mathrm{~s}, 55^{\circ} \mathrm{C}$ for $30 \mathrm{~s}$, and $72{ }^{\circ} \mathrm{C}$ for $45 \mathrm{~s}$, and a final extension at $72{ }^{\circ} \mathrm{C}$ for $\left.10 \mathrm{~min}\right)$ using primers 515F ( $5^{\prime}$-GTGCCAGC MGCCGCGG-3') and 907R (5'-CCGTCAATTCMTTTRAGTTT-3') (Xiong et al., 2012). A barcode and adapter were incorporated between the adapter and the forward primers. PCR reactions were performed in triplicate in $20-\mu \mathrm{L}$ reaction mixtures containing $4 \mu \mathrm{L}$ of $5 \times$ FastPfu Buffer, $2 \mu \mathrm{L}$ of $2.5 \mathrm{mM}$ dNTPs, $0.8 \mu \mathrm{L}$ of each primer $(5 \mu \mathrm{M}), 0.4 \mu \mathrm{L}$ of FastPfu Polymerase, $10 \mathrm{ng}$ of template DNA, and sterile double-distilled $\mathrm{H}_{2} \mathrm{O}$.

\subsection{Illumina MiSeq sequencing}

A mixture of equal quantities of the three PCR products from each sample was extracted from $2 \%$ agarose gels, purified using an AxyPrep DNA Gel Extraction Kit (Axygen Biosciences, Union City, CA, USA), and quantified using a QuantiFluor-ST fluorometer (Promega, Madison, WI, USA), following the standard PCR procedure. The purified amplicons were pooled in equimolar amounts, detected with a NanoDrop 2000 spectrophotometer and then paired-end sequenced on an Illumina MiSeq platform.

\subsection{Processing the sequencing data}

Raw fastq files were demultiplexed and quality-filtered using QIIME (V 1.17) software (Caporaso et al., 2010) with the following criteria: (I) 250 bp reads were truncated at sites that received an average quality score $<20$ over a 10 -bp sliding window, and truncated reads that were $<50 \mathrm{bp}$ were discarded; (II) exact barcode matching, two nucleotide mismatch in primer matching, reads containing ambiguous characters were removed; and (III) only sequences that had greater than a $10 \mathrm{bp}$ overlap were assembled according to their overlap sequence. Reads that could not be assembled were discarded.

Chimeras were detected using the UCHIME algorithm, and the high-quality sequences were grouped into operational taxonomic units (OTUs), at a 97\% sequence similarity threshold, using UPARSE (V 7.1) software (Edgar, 2013). RDP Classifier software (Wang et al., 2007) was used to analyze the phylogenetic affiliations of the $16 \mathrm{~S}$ rRNA gene sequences against the SILVA (SSU119) 16S rRNA database (Quast et al., 2013) at a confidence threshold of more than $70 \%$. 


\subsection{Multivariate analysis}

Microbial community richness indices (Ace and Chao) and diversity indices (Shannon and Simpson estimators) were calculated using Mothur (V 1.30.1) software (Schloss et al., 2009). The R language platform was used to visualize microbial diversity and abundance datasets from the various samples. Canonical correlation analysis (CCA) was used to test the relationship between environmental variables and microbial diversity using Canoco (V 5.0) software. The sampling and analyzing methods are shown in Fig. 1.

\section{Results and discussion}

\subsection{Characterization of soil and waste samples}

The physicochemical properties of the studied samples are shown in Table 1 . The moisture contents of the cover soil at Site1 and Site- 2 were $16.3 \%$ and $18.7 \%$, respectively, whereas the moisture content of the stored waste increased with storage depth. The moisture content of the stored waste at Site-1 (18.3-28.8\%) was lower than that at Site-2 (31.7-35.6\%). The $\mathrm{pH}$ of the cover soil and the stored waste were alkaline, and the $\mathrm{pH}$ values of the cover soil (7.8 and 7.9) were lower than those of the stored waste (7.88.6). The EC of the stored waste was 1.6-20.0 times higher than that of the cover soil ( 5.4 and $6.7 \mathrm{~ms} / \mathrm{cm}$ ). The EC of the waste at Site-1 was also significantly higher at all depths (1.6-9.9 times) compared to the waste at Site-2, indicating more soluble salt content in the Site- 1 samples. The highest EC was found at depths of $0-60 \mathrm{~cm}$ in the stored waste. CEC was not different in the cover soil and stored waste, ranging from 14.6 to $25.2 \mathrm{cmol} / \mathrm{kg}$. The organic matter content in the stored waste (101.0-186.4 g/kg) was $18-38$ times that in the cover soil $(5 \mathrm{~g} / \mathrm{kg})$. Similarly, the total carbon (TC), total nitrogen (TN), and $\mathrm{NH}_{4}^{+}-\mathrm{N}$ contents of the stored waste were 31-118 times, 8-30 times, and 15-39 times higher than those of the cover soil, respectively. The highest levels of organic matter and TC and TN contents in stored waste were observed at a depth of $120-150 \mathrm{~cm}$ at Site-1 and at a depth of $30-60 \mathrm{~cm}$ at Site- 2 .

More than $70 \%$ of the stored waste was a soil-like material, which was formed by complex biochemical reactions in the organic waste over an extended period. Therefore, the organic matter, TC, and TN contents of the stored waste were comparatively higher than those of the cover soil. Similar to previous characterization studies on stored waste in landfills, the physicochemical properties of the stored waste varied with storage depth; however, these depth differences were rarely statistically significant (Zhou et al., 2015a,b). EC may strongly affect microbial activities in landfills. In this study, the EC of stored waste at Site- 1 was similar to that reported previously (Zhou et al., 2015a,b); however, the EC was lower at Site-2.

\subsection{Richness and diversity of the microbial community}

The richness and diversity of the microbial community in the landfill are shown in Fig. 2. In total, 648,809 effective sequences with an average length of 395.46 bp were obtained from highthroughput sequencing, and the reads for each sample ranged from 31,831 to 54,233 . A total of 2139 OTUs were assigned, and the sequencing coverage rate exceeded $99 \%$ (microbial population) for each sample, indicating that exhaustive information on the microbial community was obtained by MiSeq. An average of $1036 \pm 219$ OTUs was identified in stored waste, and the minimum values were 891 at Site- 1 and 818 at Site-2, both at a depth of 60$90 \mathrm{~cm}$. The Ace and Chao indices were used to show the richness of the microbial community. The average Ace and Chao index values for the microbial community in stored waste were 500 and 496, respectively, which were 2.5-2.6 times those in cover soil, indicating higher microbial richness in stored waste. A similar trend was evident in the Shannon and Simpson diversity indices (higher Shannon and lower Simpson values reflect higher microbial diversity). The average Shannon and Simpson values for the microbial community in stored waste were 4.6 and 0.05 , which were $154 \%$ and $36 \%$ higher than those in cover soil, respectively, indicating higher microbial diversity in the stored waste. Interestingly, the microbial richness and diversity indicators showed a cross-depth trend: the Shannon and Simpson indices increased with storage depth, whereas the Ace and Chao indices decreased from the top $(0-60 \mathrm{~cm})$ to the middle layer $(60-90 \mathrm{~cm})$ and then increased in the bottom layer $(90-150 \mathrm{~cm})$.

The sequencing coverage rates in this study were higher than those obtained in a previous landfill microbial study (Song et al., 2015a), in which 454 pyrosequencing was used, indicating that a high-resolution microbial profile can be obtained by applying the MiSeq method. The richness and diversity of the microbial community in the stored waste were higher than those in the cover soil owing to the higher organic and nutrients contents in the stored waste. Changes in oxygen content may have resulted in the increased microbial diversity with storage depth. As oxygen content decreased with storage depth, the types of facultative anaerobic and anaerobic organisms likely increased. Microbe richness was lowest in the middle layer of stored waste, although the carbon and nutrient contents were moderate in all layers, indicating that the richness of the microbial community across depths in the stored waste may be affected by other environmental factors.

\subsection{Microbial taxonomic analysis at the phylum level}

High-throughput sequencing revealed the diversity of the microbial community in different samples at the phylum level (Fig. 3). These data have been uploaded to the National Center for Biotechnology Information Database (No. SRR5131582). We

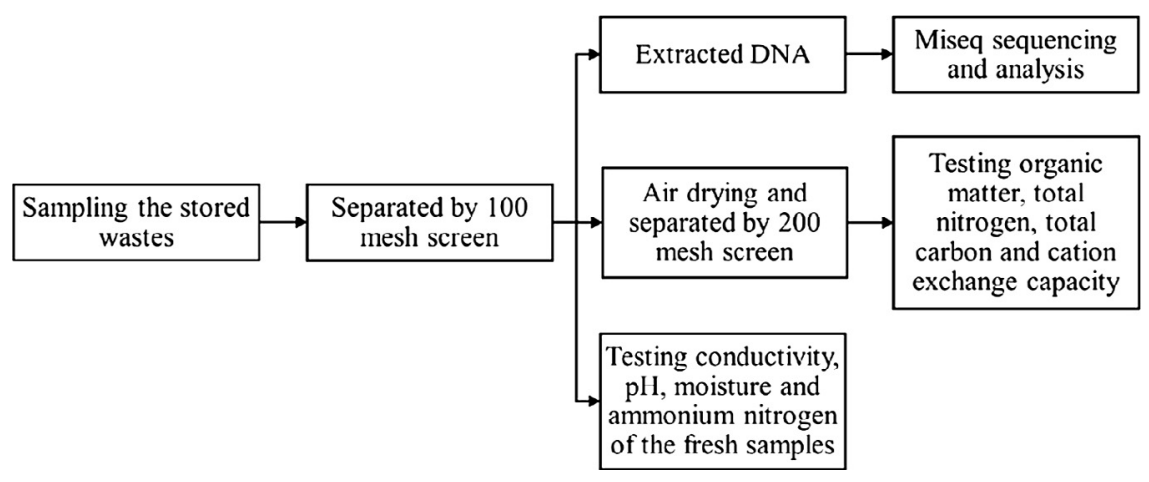

Fig. 1. Sampling and analysis methods. 
Table 1

The physical and chemical properties of cover soil and stored waste samples.

\begin{tabular}{|c|c|c|c|c|c|c|c|c|c|}
\hline Sampling sites & Sample ID & Moisture (\%) & $\mathrm{pH}$ & $\mathrm{EC}(\mathrm{ms} / \mathrm{cm})$ & $\mathrm{CEC}(\mathrm{cmol} / \mathrm{kg})$ & Organic matter $(\mathrm{g} / \mathrm{kg})$ & TC (\%) & $\mathrm{TN}(\%)$ & $\mathrm{NH}_{4}^{+}-\mathrm{N}(\mathrm{mg} / \mathrm{kg})$ \\
\hline \multirow[t]{6}{*}{ Site-1 } & $1 \_0$ & 18.7 & 7.8 & 5.4 & 19.5 & 4.9 & 0.17 & 0.03 & 9.86 \\
\hline & $1 \_3$ & 18.3 & 8.3 & 93.6 & 19.3 & 101.0 & 5.22 & 0.25 & 267.16 \\
\hline & 1_6 & 20.4 & 8.5 & 108.5 & 16.8 & 102.0 & 5.22 & 0.27 & 180.09 \\
\hline & 1_9 & 17.1 & 8.5 & 107.0 & 14.6 & 103.5 & 5.97 & 0.29 & 142.56 \\
\hline & 1_12 & 27.5 & 8.3 & 85.9 & 16.7 & 134.6 & 8.96 & 0.44 & 279.25 \\
\hline & 1_15 & 28.8 & 8.3 & 87.4 & 20.3 & 127.4 & 9.03 & 0.42 & 243.79 \\
\hline \multirow[t]{6}{*}{ Sites-2 } & $2 \_0$ & 16.3 & 7.9 & 6.7 & 20.3 & 5.7 & 0.11 & 0.02 & 9.33 \\
\hline & $2 \_3$ & 33.3 & 8.1 & 54.4 & 23.4 & 146.4 & 11.61 & 0.51 & 189.22 \\
\hline & $2 \_6$ & 31.7 & 7.8 & 33.5 & 22.3 & 186.4 & 13.07 & 0.60 & 381.05 \\
\hline & $2 \_9$ & 34.6 & 8.5 & 11.0 & 25.2 & 127.9 & 9.00 & 0.39 & 211.34 \\
\hline & 2_12 & 35.1 & 8.6 & 18.1 & 21.9 & 115.5 & 7.06 & 0.29 & 193.28 \\
\hline & $2 \_15$ & 35.6 & 8.5 & 22.3 & 21.1 & 138.5 & 8.84 & 0.36 & 202.89 \\
\hline
\end{tabular}
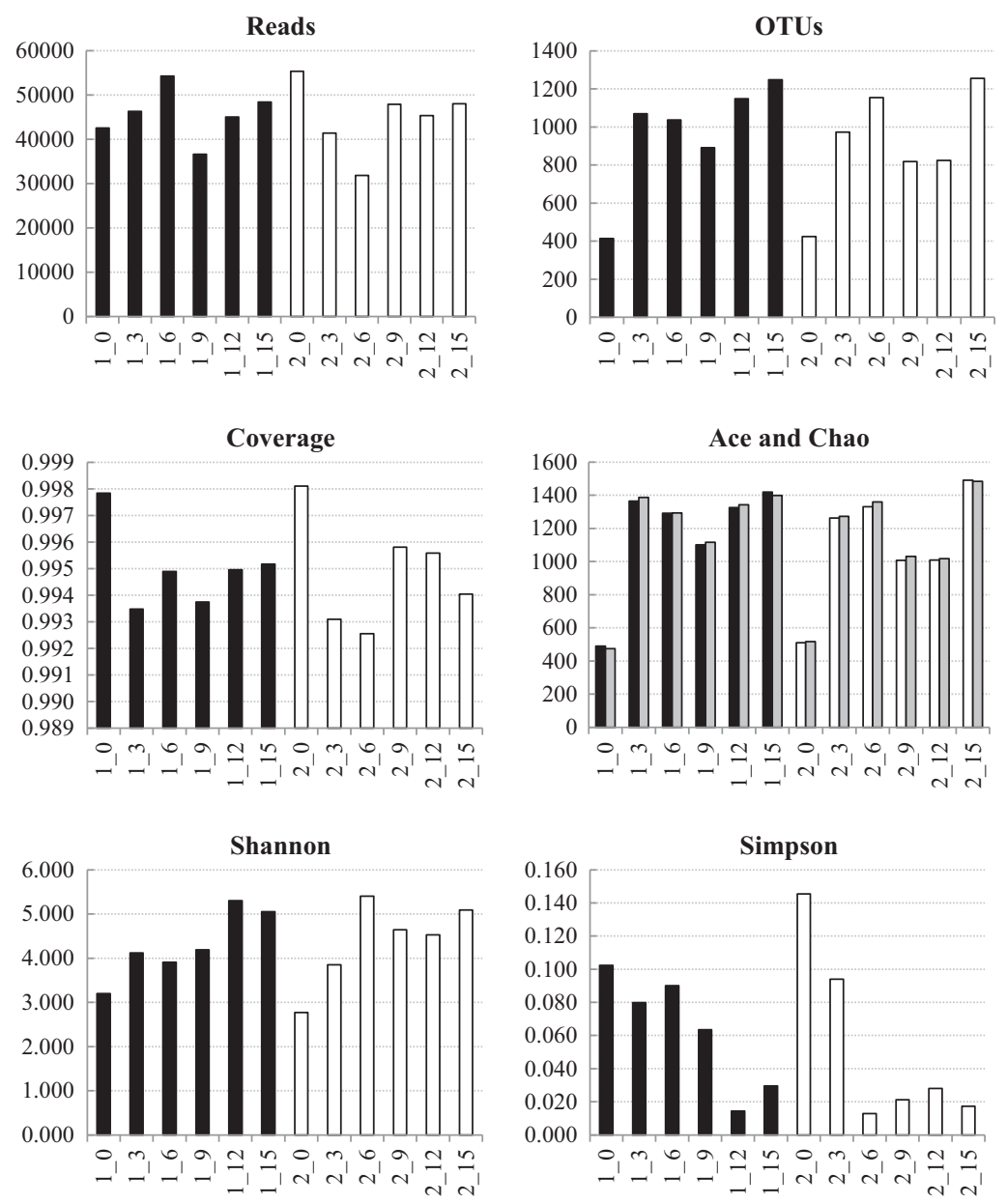

Fig. 2. Abundance and diversity of the microbial community in different samples.

found 14 phyla (each relative abundance $>1 \%$ ) using MiSeq sequencing, whereas only five phyla were detected using the TRFLP method in a previous study (Sawamura et al., 2010).

The diversity of the microbial community in stored waste was significantly higher than that in the cover soil. The phyla Proteobacteria, Bacteroidetes, Firmicutes, Spirochaetes, DeinococcusThermus, Actinobacteria, Synergistetes, Thermotogae, and Tenericutes were detected in all 12 samples collected from the two sites in the landfill. Firmicutes (46.61\%), Proteobacteria (23.84\%), and Bacteroidetes $(14.29 \%)$ were the most dominant phyla in the landfill.
These phyla have also been observed in leachate, sludge (Qiu et al., 2013), and alkaline lake sediment (Xiong et al., 2012). In particular, the abundance of Firmicutes in cover soil was similar those in samples 1_0 (33.54\%) and 2_0 (41.81\%), whereas the number of Firmicutes decreased initially in the stored waste, then increased along with the depth, and was most abundant at a depth of $30 \mathrm{~cm}$.

Firmicutes is the most dominant phylum in landfills (Krishnamurthi and Chakrabarti, 2013; Van Dyke and McCarthy, 2002). Firmicutes are cellulose-degrading bacteria and are believed 


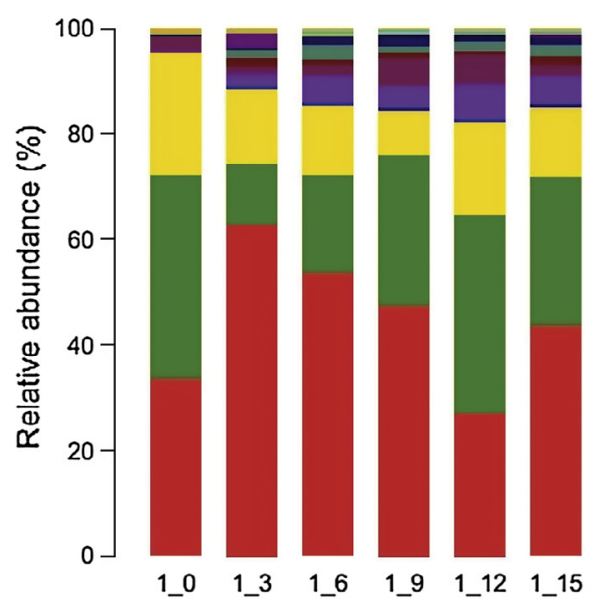

a

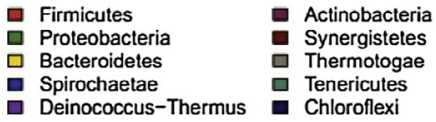

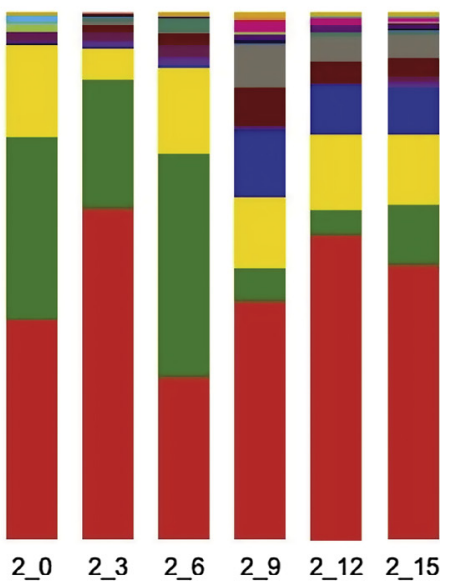

$b$

- Bacteria_unclassified

$\square$ Planctomycetes

- Candidate_division_JS1

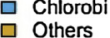

Fig. 3. Relative read abundance of different microbial community structures at the phylum level in different samples. (a) Samples from Site-1; (b) samples from Site-2.
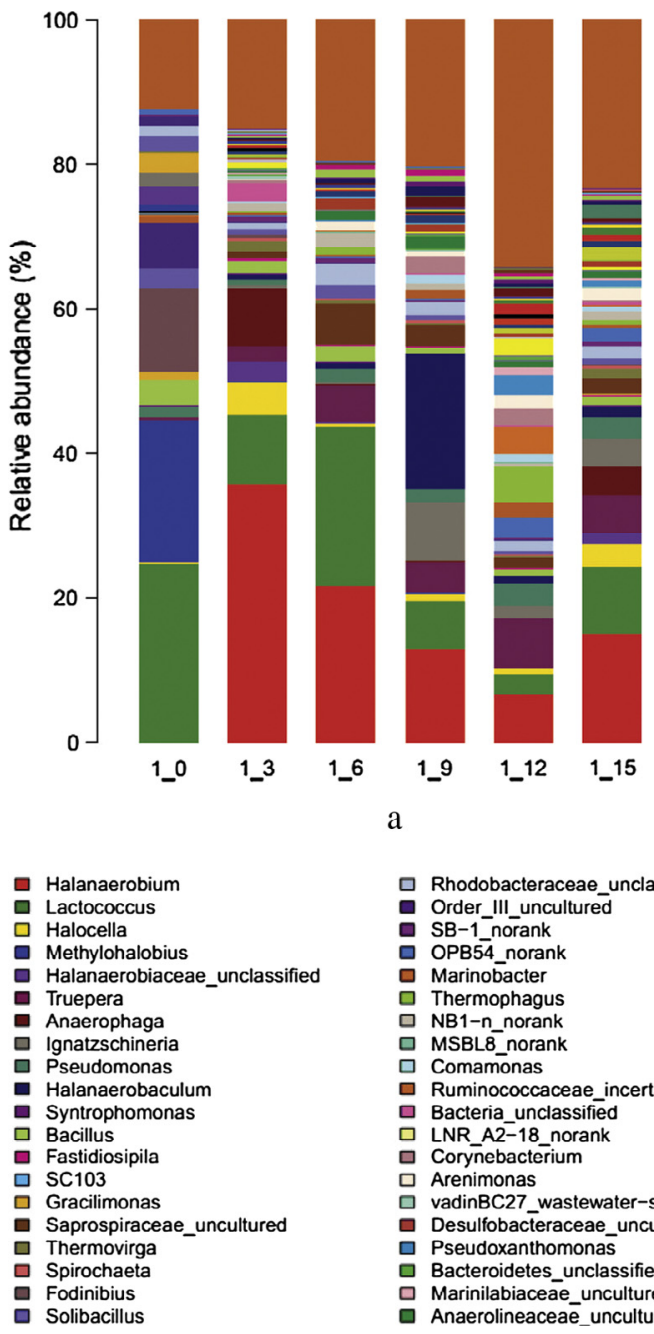

$\square$ Rhodobacteraceae_unclassified

- Order_III_uncultured

- SB-1_norank

口 OPB54_norank

- Marinobacter

Thermophagus

a NB1-n_norank

a MSBL8_norank

a Comamonas

- Ruminococcaceae_incertae_sedis

- Bacteria_unclassified

$\square$ LNR_A2-18_norank

- Corynebacterium

$\square$ Arenimonas

vadinBC27_wastewater-sludge_group

Desulfobacteraceae uncultured

$\square$ Pseudoxanthomonas

ㅁ Pseudoxanthomonas

ㅁ Bacteroidetes_unclassified

- Anaerolineaceae_uncultured
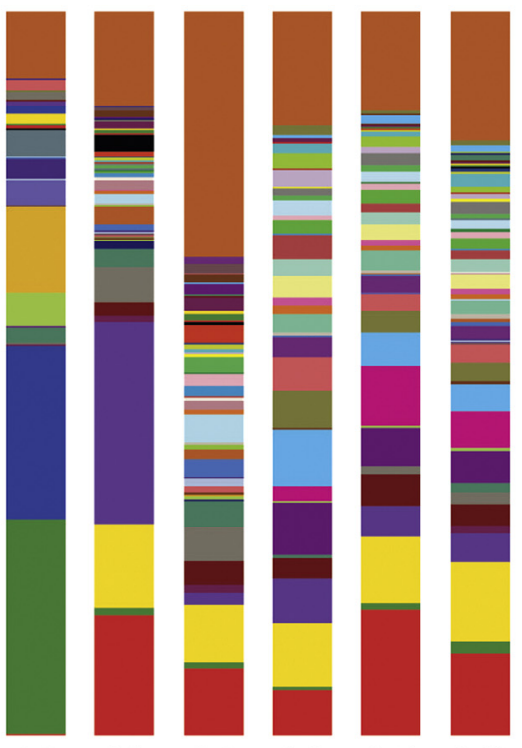

2_o

2_3

b

$\square$ Treponema

Methylohalomonas

- Proteiniphilum

- Christensenellaceae_uncultured

- Marinilabiaceae_unclassified

$\square$ Syntrophaceticus

- NS9_marine_group_norank

口 Candidate_division_JS1_norank

․ Ruminococcaceae_unclassified

ㅁ Pusillimonas

- Propionibacteriaceae_uncultured

口 Petrimonas

- Marinobacterium

- Marinobacterium

Tepidimicrobium

$\square$ Planctomyces

- Arthrobacter

- Methylophaga

- Aminobacterium

Fig. 4. Relative read abundance of different microbial community structures at the genus level in different samples. (a) Samples from Site-1; (b) samples from Site-2.

to play an important role in the anaerobic and methanogenic phases of refuse decomposition in landfills (Song et al., 2015b). The phyla Proteobacteria and Bacteroidetes are thought to play important roles in organic matter degradation and the carbon cycle (Newton et al., 2011). Gammaproteobacteria, Alphaproteobacteria, and Betaproteobacteria were detected in all samples. 


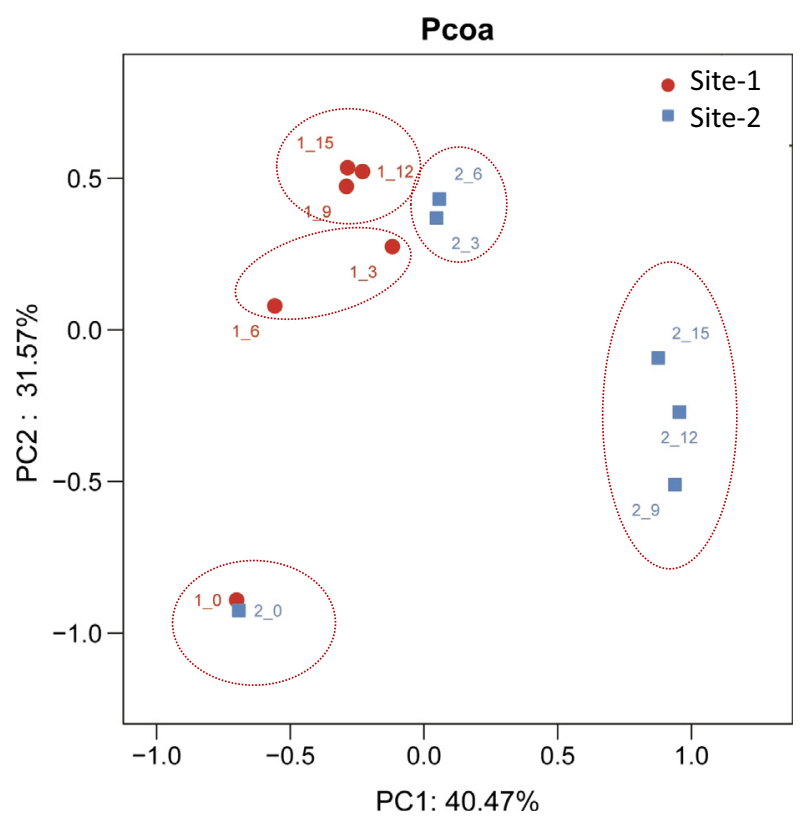

Fig. 5. Principal coordinates analysis $(\mathrm{PCoA})$ of the microbial community structure.
The phyla Deinococcus-Thermus and Actinobacteria were subdominant at Site-1. Deinococcus-Thermus was previously found in both compost (Partanen et al., 2010) and a landfill (Song et al., 2015a). Actinobacteria is a phylum capable of accelerating biodegradation and was also a subdominant group in the microbial community at Site- 1 . The lower organic matter content at Site- 1 compared with that at Site-2 may have been due to the biodegrading capacity of Actinobacteria (Table 1). The phyla Spirochaetae, Thermotogae, and Synergistetes were subdominant in the stored waste at a depth of 90-120 cm in Site-2. The phylum Spirochaetae, composed primarily of anaerobic heterotrophic microorganisms, was previously detected in landfills and in an anaerobic dynamic membrane bioreactor (Köchling et al., 2015; Xie et al., 2014). Synergistetes has been observed previously in anaerobic digestion reactors and may affect the fermentation of proteins and sugars during anaerobic processes (Morita et al., 2011; Tang et al., 2011).

\subsection{Microbial taxonomic analysis at the genus level}

The dominant microbial genera in the landfill included Halanaerobium, Lactococcus, Halocella, Methylohalobius, Truepera, Ignatzschineria, Pseudomonas, Bacillus, Syntrophomonas, Fastidiosipila, and Spirochaeta (Fig. 4). We are the first to report the predominance of the genera Halanaerobium and Ignatzschine-

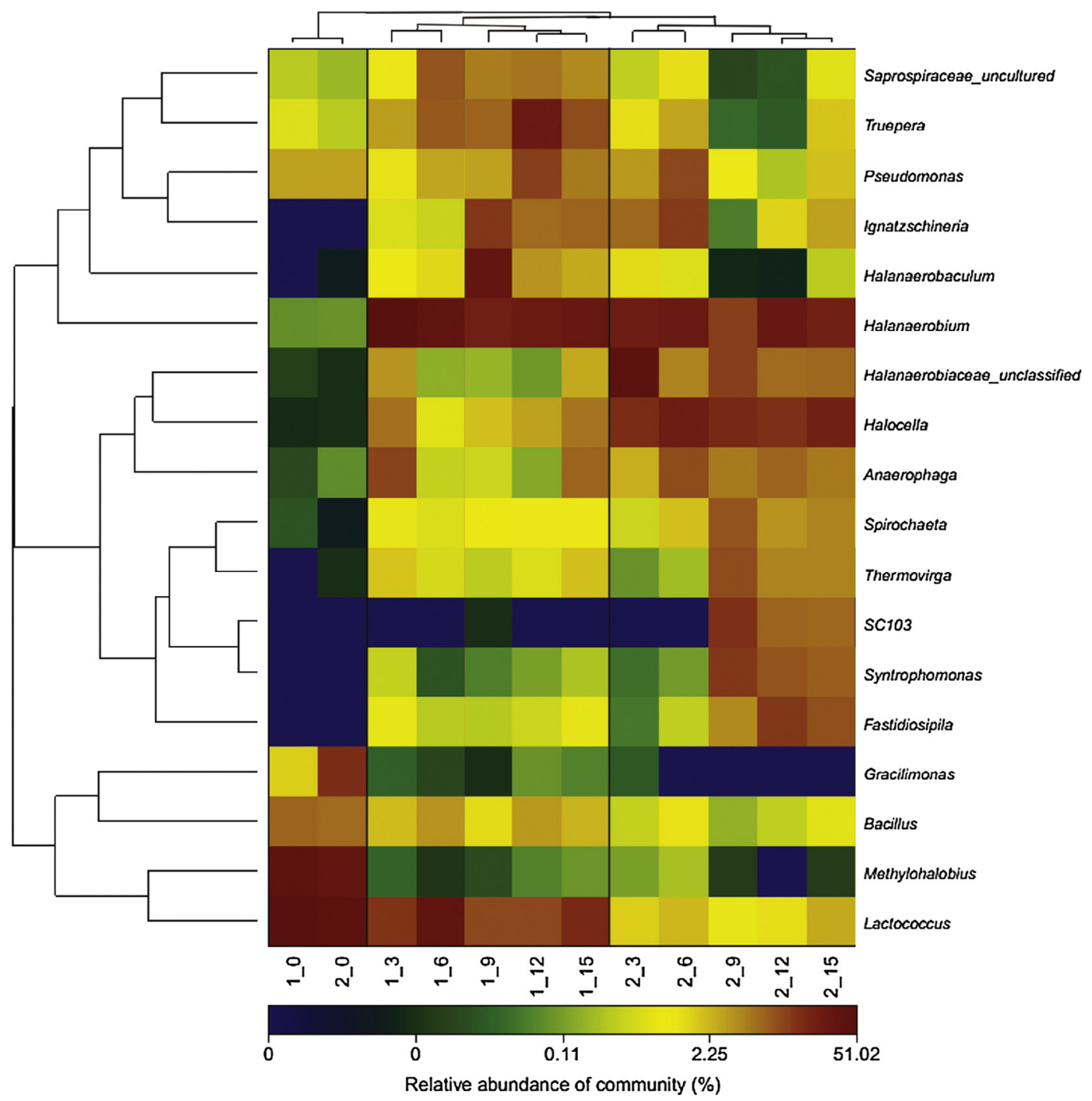

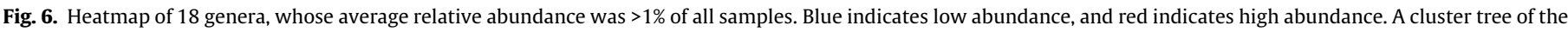
18 genera is shown on the left. (For interpretation of the references to colour in this figure legend, the reader is referred to the web version of this article.) 
ria in a landfill. Halanaerobium are anaerobic bacteria that produce hydrogen and were abundant in stored waste. As storage depth increased, the number of Halanaerobium first decreased and then increased. The abundance of Halanaerobium was lowest (6.28$6.74 \%$ ) in samples taken from a depth of $60-120 \mathrm{~cm}$, which also had a lower EC, indicating that Halanaerobium may grow better with higher salt content. Ignatzschineria was not found in cover soil, but was frequently found in stored waste; in particular, sample 1_9 had the highest abundance of Ignatzschineria (7.87\%).

Two newly found genera, Halanaerobium and Ignatzschineria, could be helpful for examining the biological processes in landfills. Halanaerobium are hydrolytic bacteria that ferment complex organic matter and produce intermediary metabolites for other microbial groups, such as sulfate-reducing and methanogenic bacteria (Ivanova et al., 2011). Halanaerobium are highly enriched in lactateand glucose-fed bioreactors (Zhou et al., 2015b). Ignatzschineria, formerly called Schineria, is a Gram-negative bacterial genus in class Gammaproteobacteria, (Gupta et al., 2011). Juteau et al. (2004) reported that Ignatzschineria was the second most abundant species in an aerobic thermophilic sequencing batch reactor; however, its function in the microbial community remains unclear.

Lactococcus and Methylohalobius were dominant in cover soil samples. The abundance of Methylohalobius was $19.76 \%$ in sample $1 \_0$ and $23.99 \%$ in sample 2_0. Lactococcus is not only capable of degrading lignocelluloses but can also reduce humic acids (Benz et al., 1998). Methylohalobius are aerobic methane-oxidizing bacteria, with a crucial role in mitigating methane emissions from landfills (Nikiema et al., 2007).

Furthermore, the genus Pseudomonas has been widely detected in landfills and other contaminated environments (Gomez et al., 2011; Li et al., 2011). Pseudomonas are well-known pollutantdegrading bacteria that utilize a wide range of polycyclic aromatic hydrocarbons as their sole carbon source (Loick et al., 2009) and have roles in organic matter degradation (Horel et al., 2015) and denitrification (Lalucat et al., 2006). Bacillus are frequently found in landfills where they degrade cellulose (Westlake et al., 1995) and oxidize polycyclic aromatic hydrocarbons (Zeng et al., 2016) and chromium (Desai et al., 2008). Truepera are mostly found in alkaline, moderately saline, and high temperature habitats (Ivanova et al., 2011) and are relatively abundant in agricultural waste (Covino et al., 2016). Halocella, which have previously been detected in landfills (Simankova et al., 1993), may be important in cellulose degradation, acid production, and methane production (Tang et al., 2011).

Syntrophomonas, Fastidiosipila, Spirochaeta, and Thermovirga were abundant in the middle and bottom layers at Site-2. The abundance of these genera ranged from 4.56 to $8.30 \%$ at a depth of 60 $150 \mathrm{~cm}$. As most of these genera are anaerobes, they can survive in the deep layer in the absence of oxygen. The genera Syntrophomonas and Fastidiosipila belong to the Clostridia class, which are efficient hydrogen producers (Kim et al., 2014). Syntrophomonas produce hydrogen and form a syntrophic relationship with hydrogen-using bacteria, such as Methanospirillum (Toumi et al., 2015). Fastidiosipila, a Gram-positive genus, was one of the most abundant genera found in a membrane bioreactor for treating landfill leachate (Xie et al., 2014). Spirochaeta may help to enhance cellulose biodegradation of landfill leachate in symbiosis with Clostridium species (Pohlschroeder et al., 1994). Most bacteria from the genus Thermovirga are thermophilic and capable of utilizing carbohydrates, proteins, amino acids, and organic acids (Li et al., 2015).

\subsection{Correlations between microbial diversity and environmental factors}

Principal coordinates analysis ( $\mathrm{PCOA})$ is a non-constrained data dimensionality reduction method that was used to further analyze

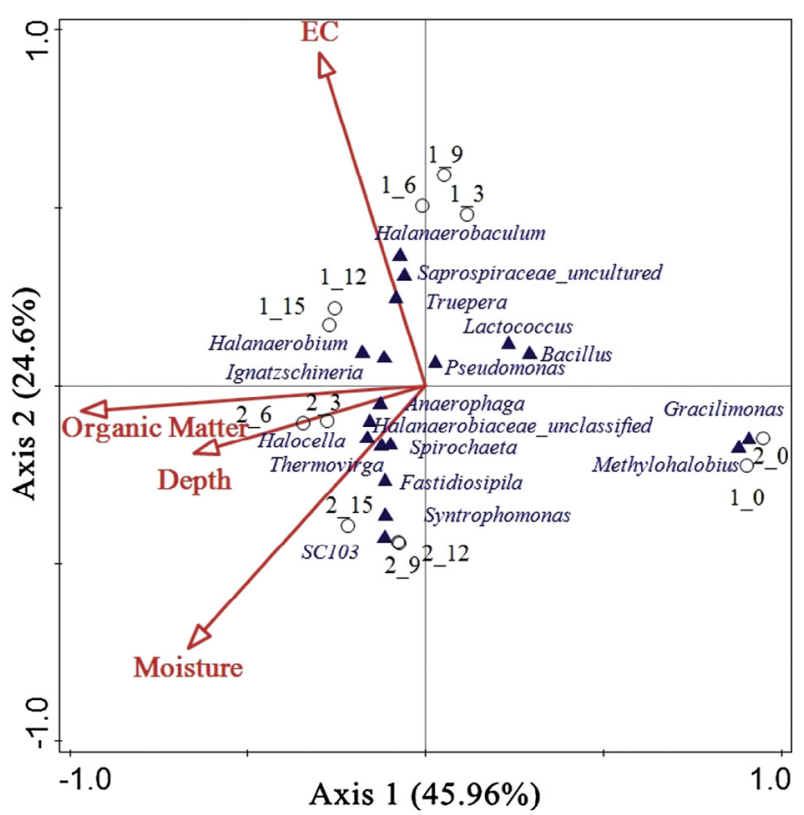

Fig. 7. Canonical correlation analysis (CCA) ordination triplot of the microbial community composition and physicochemical properties within the landfill. Blue triangles represent different genera, and circles represent the different samples. (For interpretation of the references to colour in this figure legend, the reader is referred to the web version of this article.)

the environmental factors affecting the microbial community (Fig. 5). The amount of variance accounted by the two principle components was $40.7 \%$ and $31.6 \%$. According to the PCoA analysis, the 12 samples were divided into four groups: (1) samples $1 \_0$ and 2_0; (2) samples 2_9, 2_12, and 2_15; (3) samples 2_3 and 2_6; and (4) samples 1_3, 1_6, 1_9, 1_12, and 1_15.

The clustering of the 18 genera with an average relative abundance $>1 \%$ in all samples is shown in Fig. 6 (supporting data are shown in Table SI). Samples 1_3 and 1_6 had a significantly lower abundance of Halanaerobium and Ignatzschineria than samples 1_9, 1_12, and 1_15, which separated samples 1_3 and 1_6 from the other three samples at Site-1. Furthermore, samples from Site-2 were clustered into two groups due to a high abundance of Syntrophomonas, Fastidiosipila, Spirochaeta, and Thermovirga in samples 2_9, 2_12, and 2_15, whereas samples 2_3 and 2_6 exhibited low abundance. The microbial communities exhibited similar clustering trends, suggesting that the 18 dominant genera were highly representative of the major microbes in the community samples. Fig. 6 reveals that microbial community structure varied in the samples from Site- 1 and Site-2 but that the microbial community structures in the cover soil from the two study sites were similar. The microbial community structure in stored waste at depths of 90,120 , and $150 \mathrm{~cm}$ was homogeneous, whereas it was similar at depths of 30 and $60 \mathrm{~cm}$.

To further estimate the effects of environmental factors on microorganisms, the 18 genera with an average relative abundance $>1 \%$ in all samples were analyzed by detrended correspondence analysis using Canoco 5.0. A CCA sequencing diagram was developed using EC, soil organic matter levels, moisture content, and soil depth as environmental factors (Fig. 7). The analysis demonstrated that the environmental factors were strongly correlated with the microbial structure $(p<0.01)$. As also shown in Fig. 7, 45.96\% of the information corresponded to the first sequencing axis, and $24.6 \%$ corresponded to the second axis. The 18 genera were all located in the vicinity of coordinate origin, excluding Methylohalobiusa and Gracilimonas, because these two genera were present only in cover soil. 
The arrow lengths in Fig. 7 indicate that EC and organic matter and water content had significant effects on the microbial community structure. Other studies have demonstrated that moisture content is a dominant factor affecting the microbial community structure in landfills (Townsend et al., 1996). Moisture content was positively correlated with organic matter and soil depth. Eight genera, including Halocella in the third quadrant, were strongly positively correlated with moisture content. Five genera, including Halanaerobium in the second quadrant and Pseudomonas in the first quadrant, were primarily positively affected by EC. Methylohalobius and Gracilimonas had no clear associations with any of the environmental factors.

\section{Conclusions}

MiSeq methodology was applied to provide a high-resolution profile of the microbial community in a landfill. Microbial richness and abundance were significantly higher in stored waste than in the cover soil of the landfill. The microbial diversity analysis revealed that more microbial richness was found in the top and bottom layers of stored waste; however, less richness was found lower in the middle layer. According to our survey, Firmicutes, Proteobacteria, and Bacteroidetes were the most dominant phyla, whereas Halanaerobium, Lactococcus, Methylohalobius, Ignatzschineria, Syntrophomonas, Fastidiosipila, and Spirochaeta were the dominant genera. Halanaerobium and Ignatzschineria were reported in a landfill for the first time. The microbial diversity and structure in the landfill were affected by the physicochemical properties as well as the storage depths of the waste. EC and organic matter and moisture contents were the dominant factors affecting microbial diversity and structure. The dominant genus, which primarily consisted of halotolerant bacteria, may be ascribed to the high salinity in the stored waste.

\section{Conflict of interest}

The authors declare that they have no conflict of interest.

\section{Acknowledgements}

This work was financially supported by the National Natural Science Foundation of China - China (Nos. 31570019, J1310005, 71533004), National Key Research and Development Program (2016YFC0502804), and Natural Science Foundation of Jiangsu Province - China (BK20151302). We were grateful to the reviewers and English native editors for helping us improve the paper.

\section{Appendix A. Supplementary material}

Supplementary data associated with this article can be found, in the online version, at http://dx.doi.org/10.1016/j.wasman.2017.04. 023.

\section{References}

Bareither, C.A., Wolfe, G.L., McMahon, K.D., Benson, C.H., 2013. Microbial diversity and dynamics during methane production from municipal solid waste. Waste Manage. 33, 1982-1992.

Benz, M., Schink, B., Brune, A., 1998. Humic acid reduction by Propionibacterium freudenreichii and other fermenting bacteria. Appl. Environ. Microbiol. 64, 4507-4512.

Caporaso, J.G., Kuczynski, J., Stombaugh, J., Bittinger, K., Bushman, F.D., Costello, E.K., Fierer, N., Pena, A.G., Goodrich, J.K., Gordon, J.I., Huttley, G.A., 2010. QIIME allows analysis of high-throughput community sequencing data. Nat. Methods 7, 335-336.

Covino, S., Fabianová, T., Křesinová, Z., Čvančarová, M., Burianová, E., Filipová, A., Vořísková, J., Baldrian, P., Cajthaml, T., 2016. Polycyclic aromatic hydrocarbons degradation and microbial community shifts during co-composting of creosotetreated wood. J. Hazard. Mater. 301, 17-26.

Desai, C., Jain, K., Madamwar, D., 2008. Evaluation of in vitro Cr (VI) reduction potential in cytosolic extracts of three indigenous Bacillus sp. isolated from $\mathrm{Cr}$ (VI) polluted industrial landfill. Bioresour. Technol. 99, 6059-6069.

Dong, J., Ding, L.J., Wang, X., Chi, Z.F., Lei, J.S., 2015. Vertical profiles of community abundance and diversity of anaerobic methanotrophic archaea (ANME) and bacteria in a simple waste landfill in North China. Appl. Biochem. Biotechnol 175, 2729-2740.

Edgar, R.C., 2013. UPARSE: highly accurate OTU sequences from microbial amplicon reads. Nat. Methods 10, 996-998.

Fernandes, H., Viancelli, A., Martins, C.L., Antonio, R.V., Costa, R.H.R., 2013. Microbial and chemical profile of a ponds system for the treatment of landfill leachate. Waste Manage. 33, 2123-2128.

Gieg, L.M., Fowler, S.J., Berdugo-Clavijo, C., 2014. Syntrophic biodegradation of hydrocarbon contaminants. Curr. Opin. Biotechnol. 27, 21-29.

Glenn, T.C., 2011. Field guide to next-generation DNA sequencers. Mol. Ecol. Resour 11, 759-769.

Gomez, A.M., Yannarell, A.C., Sims, G.K., Cadavid-Restrepo, G., Herrera, C.X.M., 2011 Characterization of microbial diversity at different depths in the Moravia Hill landfill site at Medellín, Colombia. Soil Biol. Biochem. 43, 1275-1284.

Gupta, A.K., Dharne, M.S., Rangrez, A.Y., Verma, P., Ghate, H.V., Rohde, M., Patole, M. S., Shouche, Y.S., 2011. Ignatzschineria indica sp. nov. and Ignatzschineria ureiclastica sp. nov., isolated from adult flesh flies (Diptera: Sarcophagidae). Int. J. Syst. Evol. Microbiol. 61, 1360-1369.

Horel, A., Mortazavi, B., Sobecky, P.A., 2015. Input of organic matter enhances degradation of weathered diesel fuel in sub-tropical sediments. Sci. Total Environ. 533, 82-90.

IPCC, 2014. Climate Change 2014: Mitigation of Climate Change.

Ivanova, N., Rohde, C., Munk, C., Nolan, M., Lucas, S., Del Rio, T.G., Tice, H. Deshpande, S., Cheng, J.F., Tapia, R., Han, C., 2011. Complete genome sequence of Truepera radiovictrix type strain (RQ-24 T). Stand. Genom. Sci. 4, 91-99.

Juteau, P., Tremblay, D. Villemur, R., Bisaillon, J.G., Beaudet, R, 2004. Analysis of the microbial community inhabiting an aerobic thermophilic sequencing batch reactor (AT-SBR) treating swine waste. Appl. Microbiol. Biotechnol. 66, 115 122.

Köchling, T., Sanz, J.L., Gavazza, S., Florencio, L., 2015. Analysis of microbial community structure and composition in leachates from a young landfill by 454 pyrosequencing. Appl. Microbiol. Biotechnol. 99, 1-12.

Kim, S., Bae, J., Choi, O., Ju, D., Lee, J., Sung, H., Park, S., Sang, B.I., Um, Y., 2014. A pilot scale two-stage anaerobic digester treating food waste leachate (FWL): performance and microbial structure analysis using pyrosequencing. Process Biochem. 49, 301-308.

Krishnamurthi, S., Chakrabarti, T., 2013. Diversity of bacteria and archaea from a landfill in Chandigarh, India as revealed by culture-dependent and cultureindependent molecular approaches. Syst. Appl. Microbiol. 36, 56-68.

Lalucat, J., Bennasar, A., Bosch, R., García-Valdés, E., Palleroni, N.J., 2006. Biology of Pseudomonas stutzeri. Microbiol. Mol. Biol. Rev. 70, 510-547.

Lee, E.H., Moon, K.E., Kim, T.G., Cho, K.S., 2014. Depth profiles of methane oxidation potentials and methanotrophic community in a lab-scale biocover. J. Biotechnol. 184, 56-62.

Li, L., He, Q., Ma, Y., Wang, X.M., Peng, X.Y., 2015. Dynamics of microbial community in a mesophilic anaerobic digester treating food waste: relationship between community structure and process stability. Bioresour. Technol. 189, 113-120.

Li, Y., Low, G.K.C., Scott, J.A., Amal, R., 2011. Microbial transformation of arsenic species in municipal landfill leachate. J. Hazard. Mater. 188, 140-147.

Loick, N., Hobbs, P.J., Hale, M.D., Jones, D.L., 2009. Bioremediation of poly-aromatic hydrocarbon (PAH)-contaminated soil by composting. Crit. Rev. Environ. Sci. Technol. 39, 271-332.

Morita, M., Malvankar, N.S., Franks, A.E., Summers, Z.M., Giloteaux, L., Rotaru, A.E. Rotaru, C., Lovley, D.R., 2011. Potential for direct interspecies electron transfer in methanogenic wastewater digester aggregates. MBio 2, 41-43.

NCCC, 2013. The People's Republic of China Second National Communication on Climate Change.

Newton, R.J., Jones, S.E., Eiler, A., McMahon, K.D., Bertilsson, S., 2011. A guide to the natural history of freshwater lake bacteria. Microbiol. Mol. Biol. Rev. 75, 14-49.

Nikiema, J., Brzezinski, R., Heitz, M., 2007. Elimination of methane generated from landfills by biofiltration: a review. Rev. Environ. Sci. Biotechnol. 6, 261-284.

Nikolskii, N.N., 1963. Soil Science. US Dept Commerce, Washington, DC, p. 240.

Partanen, P., Hultman, J., Paulin, L., Auvinen, P., Romantschuk, M., 2010. Microbial diversity at different stages of the composting process. BMC Microbiol. 10, 2010-2015.

Pohlschroeder, M., Leschine, S.B., Canale-Parola, E., 1994. Spirochaeta caldaria sp. nov., a thermophilic bacterium that enhances cellulose degradation by Clostridium thermocellum. Arch. Microbiol. 161, 17-24.

Qiu, G.L., Song, Y.H., Zeng, P., Duan, L., Xiao, S.H., 2013. Characterization of microbial communities in hybrid upflow anaerobic sludge blanket (UASB)-membrane bioreactor (MBR) process for berberine antibiotic wastewater treatment Bioresour. Technol. 142, 52-62.

Quast, C., Pruesse, E., Yilmaz, P., Gerken, J., Schweer, T., Yarza, P., Peplies, J., Glöckner F.O., 2013. The SILVA ribosomal RNA gene database project: improved data processing and web-based tools. Nucleic Acids Res. 41, 590-596.

Rhoades, J.D., 1982. Cation Exchange Capacity. Methods of Soil Analysis. Part 2. Chemical and Microbiological Properties, pp. 149-157.

Sang, N.N., Soda, S., Ishigaki, T., Ike, M., 2012. Microorganisms in landfill bioreactors for accelerated stabilization of solid wastes. J. Biosci. Bioeng. 114, 243-250. 
Sawamura, H., Yamada, M., Endo, K., Soda, S., Ishigaki, T., Ike, M., 2010. Characterization of microorganisms at different landfill depths using carbonutilization patterns and 16S rRNA gene based T-RFLP. J. Biosci. Bioeng. 109, 130-137.

Schloss, P.D., Westcott, S.L., Ryabin, T., Hall, J.R., Hartmann, M., Hollister, E.B., Lesniewski, R.A., Oakley, B.B., Parks, D.H., Robinson, C.J., Sahl, J.W., 2009. Introducing mothur: open-source, platform-independent, communitysupported software for describing and comparing microbial communities. Appl. Environ. Microbiol. 75, 7537-7541.

Simankova, M.V., Chernych, N.A., Osipov, G.A., Zavarzin, G.A., 1993. Halocella cellulolytica gen. nov., sp. nov., a new obligately anaerobic, halophilic, cellulolytic bacterium. Syst. Appl. Microbiol. 16, 385-389.

Slezak, R., Krzystek, L., Ledakowicz, S., 2015. Degradation of municipal solid waste in simulated landfill bioreactors under aerobic conditions. Waste Manage. 43, 293-299.

Song, L.Y., Wang, Y.Q., Zhao, H.P., Long, D.T., 2015a. Composition of microbial and archaeal communities during landfill refuse decomposition processes. Microbiol. Res. 181, 105-111.

Song, L.Y., Wang, Y.O., Tang, W., Lei, Y., 2015b. Microbial community diversity in municipal waste landfill sites. Appl. Microbiol. Biotechnol. 99, 7745-7756.

Stralis-Pavese, N., Sessitsch, A., Weilharter, A., Reichenauer, T., Riesing, J., Csontos, J. Murrell, J., Bodrossy, L., 2004. Optimization of diagnostic microarray for application in analysing landfill methanotroph communities under different plant covers. Environ. Microbiol. 6, 347-363.

Tang, Y.Q., Ji, P., Hayashi, J., Koike, Y., Wu, X.L., Kida, K., 2011. Characteristic microbial community of a dry thermophilic methanogenic digester: its longterm stability and change with feeding. Appl. Microbiol. Biotechnol. 91, 1447 1461.

Toumi, J., Miladi, B., Farhat, A., Nouira, S., Hamdi, M., Gtari, M., Bouallagui, H., 2015. Microbial ecology overview during anaerobic codigestion of dairy wastewate and cattle manure and use in agriculture of obtained bio-fertilisers. Bioresour. Technol. 198, 141-149.

Townsend, T., Miller, W., Lee, H.J., Earle, J., 1996. Acceleration of landfill stabilization using leachate recycle. J. Environ. Eng. 122, 263-268.

Uchida, M., Hatayoshi, H., Syuku-nobe, A., Shimoyama, T., Nakayama, T., Okuwaki, A., Nishino, T., Hemmi, H., 2009. Polymerase chain reaction-denaturing gradient gel electrophoresis analysis of microbial community structure in landfill leachate. J. Hazard. Mater. 164, 1503-1508.

USEPA, 2009. Municipal Solid Waste Generation, Recycling, and Disposal in the United States Detailed Tables and Figures for 2008.

USEPA, 2016. Inventory of U.S. Greenhouse Gas Emissions and Sinks: 1990-2014

Van Dyke, M., McCarthy, A., 2002. Molecular biological detection and characterization of Clostridium populations in municipal landfill sites. Appl. Environ. Microbiol. 68, 2049-2053.

Wang, Q., Garrity, G.M., Tiedje, J.M., Cole, J.R., 2007. Naive Bayesian classifier for rapid assignment of rRNA sequences into the new microbial taxonomy. Appl. Environ. Microbiol. 73, 5261-5267.

Wang, Z.B., Shao, S.P., Zhang, C.S., Lu, D.L., Ma, H.L., Ren, X.F., 2015, Pretreatment of vinegar residue and anaerobic sludge for enhanced hydrogen and methane production in the two-stage anaerobic system. Int. J. Hydrogen Energy 40, 4494-4501.

Westlake, K., Archer, D., Boone, D., 1995. Diversity of cellulolytic bacteria in landfill. J. Appl. Bacteriol. 79, 73-78.

Xie, Z.F., Wang, Z.W., Wang, Q.Y., Zhu, C.W., Wu, Z.C., 2014. An anaerobic dynamic membrane bioreactor (AnDMBR) for landfill leachate treatment: performance and microbial community identification. Bioresour. Technol. 161, 29-39.

Xiong, J., Liu, Y., Lin, X., Zhang, H., Zeng, J., Hou, J., Yang, Y., Yao, T., Knight, R., Chu, H., 2012. Geographic distance and pH drive microbial distribution in alkaline lake sediments across Tibetan Plateau. Environ. Microbiol. 14, 2457-2466.

Zeng, J., Zhu, Q., Wu, Y., Lin, X., 2016. Oxidation of polycyclic aromatic hydrocarbons using Bacillus subtilis CotA with high laccase activity and copper independence. Chemosphere 148, 1-7.

Zhang, D.Q., Tan, S.K., Gersberg, R.M., 2010. Municipal solid waste management in China: status, problems and challenges. J. Environ. Manage. 91, 1623-1633.

Zhang, W., Chen, L., Zhang, R., Lin, K.F., 2016. High throughput sequencing analysis of the joint effects of BDE209-Pb on soil microbial community structure. J. Hazard. Mater. 301, 1-7.

Zhou, C.B., Xu, W.Y., Gong, Z., Fang, W.J., Cao, A.X., 2015a. Characteristics and fertilizer effects of soil-like materials from landfill mining. Clean (Weinh) 43, 940-947.

Zhou, J.M., Zhou, X.M., Li, Y.G., Xing, J.M., 2015b. Microbial communities in haloalkaliphilic sulfate-reducing bioreactors under different electron donors revealed by 16S rRNA MiSeq sequencing. J. Hazard. Mater. 295, 176-184. 